\title{
El conocimiento especializado del profesor de matemáticas (MTSK)
}

Alejandra Adame Esparza y Erick del Refugio de Lira Lozano

\section{Resumen}

$\mathrm{E}$ n la actualidad, la labor docente en la educación media superior, específicamente en el área de matemáticas, es realizada por una gran variedad de profesionistas que cuentan con diversos perfiles dentro de su formación inicial. La problemática recae en que gran parte de estos profesionistas no han sido formados con un perfil docente para cubrir los requerimientos demandados por este nivel. Es por ello que la matemática educativa, en su afán de contribuir al desarrollo profesional de los docentes en activo, propone el modelo del conocimiento especializado del profesor de matemáticas (Mathematics Teacher's Specialised Knowledge, мTSк), el cual pretende hacer reflexionar acerca de los conocimientos que un profesor de matemáticas debiera poseer para desempeñar esta labor de manera especializada y en beneficio de los estudiantes. La presentación de este modelo es lo que atañe el presente artículo.

Palabras clave: profesionalización docente, matemáticas, conocimiento especializado del profesor, educación media superior, MTSK.

\section{Introducción}

Desde finales del siglo xix y principios del siglo xx las escuelas normales en México se han dedicado a la formación de los profesionistas encargados de la enseñanza, al menos en los niveles preescolar, primaria y secundaria, no así en los niveles medio superior y superior. Para estos últimos niveles, ¿cómo se forma una persona que aspira a ser profesor?; específicamente, ¿qué conocimientos son necesarios para que una persona pueda desempeñarse como profesor de matemáticas?, y ya como profesor de esta materia, ¿qué se posee y de qué se carece?

A partir del año 2012 se reconoció la obligatoriedad de la educación media superior. Esto trajo consigo una serie de nuevas áreas de oportunidad en la mejora de los procesos de enseñanza y aprendizaje. Se buscó que los estudiantes tuvieran una educación de calidad y adquirieran las herramientas básicas para el desarrollo de la vida en sociedad. Para que lo anterior sea posible llevarlo a cabo, es preciso que los actores encargados de la formación de estos estudiantes sean competentes y estén preparados para ello. Con la reforma educativa se pone en la mira al profesor y el papel que éste debería desempeñar en las aulas de clase, por lo cual se busca que esté en continua formación y actualización.

Uno de los problemas en la educación media superior, particularmente en el área de matemáticas, es que los encargados de la labor docente presentan una gran diversidad respecto a su formación inicial. Esto se manifiesta en "Tuning Latinoamérica" (Beneitone, Esquetini, González, Marty, Siufi y Wagenaar, 2007), donde se establece que esta labor es compartida por profesionistas como ingenieros, físicos, matemáticos y químicos, entre otros. El problema es que dichos perfiles, si bien "incluyen formación en matemáticas, no tienen como objetivo la enseñanza de las mismas. Lo anterior puede presentar algunas limitantes entre los perfiles y los recursos que activarán para atender los retos en su práctica docente" (Pérez, 2017: 20).

La formación inicial del profesional en la enseñanza de las matemáticas es escasa, considerando que la Asociación Nacional de Universidades e Instituciones de Educación Superior (ANUIES) refiere que de todos los programas de estudio a nivel superior que existen en el país, 13 son de licenciatura (formación inicial), están relacionados con la enseñanza y el aprendizaje de las matemáticas, existiendo además a nivel posgrado (for- 
mación continua) 26 programas dedicados al mismo fin (ANuies, 2012, citado en Hernández, Sosa y López, 2013):

Si se hace un comparativo respecto a la oferta nacional en el área de educación y pedagogía, entonces tendríamos que sólo el 5.8\% (13 de 224) de la oferta de formación inicial va dirigido a la profesionalización específica del profesor de matemáticas. Y en cuanto a la formación continua alcanza un 8.6\% (26 de 302) de la oferta nacional (Hernández, Sosa y López, 2013: 13).

\section{« En la educación media superior,} particularmente en el área de matemáticas, la labor docente presenta una gran diversidad respecto a su formación inicial.»)

\section{Desarrollo}

¿Qué hacer ante tal diversidad de formaciones? Sin lugar a duda cada profesional que imparte clases de matemáticas lo hace porque es capaz de hacerlo, pero ante tal diversidad de perfiles es inminente que se definan los conocimientos básicos y especializados que todo profesor de matemáticas debe tener para que, además de brindar una educación de vanguardia, cumpla con los perfiles deseados de las reformas actuales. La gran cantidad de enfoques, posturas y experiencias hacen de la enseñanza de las matemáticas un abanico lleno de matices, en el cual, en ocasiones, los docentes terminan enseñando sólo aquello que se les facilita, en lo que son buenos, lo que les gusta, careciendo de un criterio que ayude a homogeneizar y enriquecer lo que se enseña.

El problema tiene que ser resuelto y no existe ni el tiempo ni los recursos para cambiar a toda la planta docente existente que no cuente con perfil inicial de docente de matemáticas. Parte de la solución se encuentra en la profesionalización de los docentes. Ante este reto, la matemática educativa propone el modelo del conocimiento especializado del profesor de matemáticas (MTSK).

El modelo MTSK es una invitación a la reflexión y a la autocrítica; se considera "una propuesta teórica que modela el conocimiento núcleo del conocimiento profesional del profesor de matemáticas y es, a su vez, una herramienta metodológica que permite analizar distintas prácticas del profesor de matemáticas a través de sus categorías" (Flores, Escudero, Montes y Aguilar, 2014: 1).

Permite reflexionar acerca de lo que se posee o se debería poseer para el desempeño de la docencia en matemáticas. Este modelo considera dos grandes dominios del conocimiento: el conocimiento matemático (mathematical knowledge, $\mathrm{MK}$ ) y el conocimiento didáctico del contenido (pedagogical content knowledge, $\mathrm{PCK}$ ):

\section{Dominio del conocimiento matemático (MK)}

Comprende el conocimiento de los temas matemáticos, el de la estructura matemática y la práctica matemática. Se divide, a su vez, en tres subdominios, los cuales son caracterizados en diversas categorías:

- Conocimiento de los temas matemáticos (knowledge of topics, кот). Es el conocimiento profundo y fundamentado del contenido matemático. Las categorías en este subnivel son: fenomenología, propiedades y fundamentos, registros de representación, definiciones y procedimientos.

- Conocimiento de la estructura matemática (knowledge of the structure of mathematics, KSM). Es el conocimiento de las relaciones entre contenidos matemáticos, ya sea dentro del mismo curso o conexiones con cursos anteriores o posteriores (incluso conexiones entre temas vistos en distintos niveles educativos). "Se trata específicamente de conexiones entre temas matemáticos" (Flores et al., 2014: 5). Las categorías correspondientes a este subnivel son: conexiones de complejización, simplificación, contenidos transversales y auxiliares.

- Conocimiento de la práctica matemática (KPM). Es el conocimiento de las formas de proceder para llegar a resultados matemáticos establecidos, así como las características del trabajo matemático. Este subnivel abarca las categorías de: prácticas ligadas a la matemática general y a una temática en matemáticas.

\section{« El modelo mTSK es una invitación a la reflexión y} a la autocrítica.» 


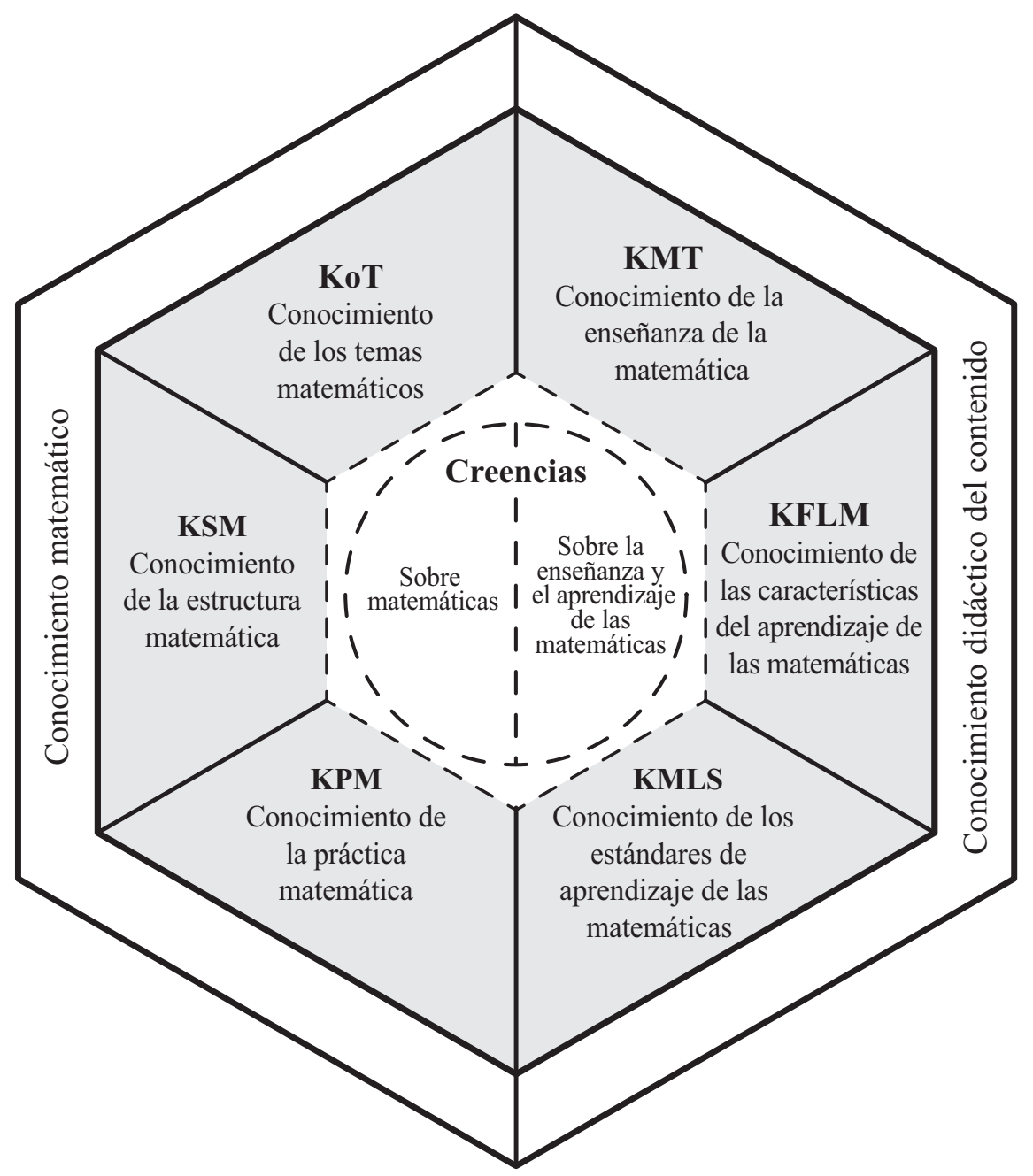

Figura 1. Esquema del modelo MTSK

Fuente: Sosa, Flores y Carrillo (2015: 175).

\section{Dominio del conocimiento didáctico del contenido (PCK)}

Es un conocimiento particular del profesor, propio de la labor de enseñanza [...]. Responde al reconocimiento de la importancia de que el profesor conozca el contenido matemático desde el punto de vista de un contenido a enseñar, desde el punto de vista de un contenido a aprender y desde una visión general de los estándares de aprendizaje que se pueden/pretenden alcanzar (Flores et al., 2014: 9).

Los subdominios en los que se divide son:

- Conocimiento de las características del aprendizaje (knowledge of features of learning mathematics, KFLM). Este subdominio se refiere a que el docente conozca y domine las distintas maneras de enseñar los contenidos matemáticos; conozca el tipo de obstáculos y dificultades que los estudiantes presentarán al momento de enfrentarse con las temáticas; advierta el pasado matemático de los estudiantes y las percepciones que guardan respecto a la materia:

Son los conocimientos sobre las características de aprendizaje inherentes al contenido matemático. Evita mirar al estudiante como el foco principal del proceso cambiando la mirada hacia el contenido matemático como objeto de aprendizaje. Esto no implica que quitemos importancia al papel del estudiante en el proceso, sino que nos interesa el conocimiento relacionado con las características de aprendizaje derivadas de su interacción con el contenido matemático y no las características del estudiante en sí mismo (Flores et al., 2014: 9). 
Sus categorías son: formas de aprendizaje, fortalezas y dificultades asociadas al aprendizaje, formas de interacción de los alumnos con el contenido matemático y concepciones de los estudiantes sobre las matemáticas (Flores et al., 2014).

- Conocimiento de la enseñanza de la matemática (knowledge of mathematics teaching, кмт). Son los conocimientos de "recursos, materiales, modos de presentar el contenido y el potencial que puede tener para la instrucción, así como el conocimiento de ejemplos adecuados para cada contenido, intención o contexto determinado" (Flores et al., 2014: 10). Las categorías de este subnivel son: formas de enseñanza, así como recursos y materiales.

- Conocimiento de los estándares de aprendizaje de las matemáticas (knowledge of mathematics learning standards, KMLs). Es el conocimiento que tiene el profesor acerca de lo que está estipulado que aprenda un estudiante del tipo educativo medio superior, al igual que el nivel conceptual con el que se espera aprenda. Entre las categorías de este subnivel están los contenidos matemáticos requeridos para enseñar, el conocimiento del nivel de desarrollo conceptual y procedimental esperado y la secuenciación de diversos temas.

El papel de las creencias y concepciones del docente es relevante en este modelo y están presentes, ya que no se debe perder de vista que éstas permean en todo momento los conocimientos y la práctica del profesor.

\section{Conclusión}

Analizar el proceso de enseñanza-aprendizaje desde el punto de vista del que enseña permite comprender la complejidad de su labor y caer en cuenta de que aprender a enseñar matemáticas implica no sólo conocer el conocimiento a enseñar, su sustento teórico, estructura y fundamentos; sino, además, conocer las diferentes maneras de abordar el tema, comprender las diversas formas de transmitirlo, conocer los recursos con los que se puede lograr la adquisición del conocimiento por parte de los estudiantes, todo esto tomando en cuenta los estándares mínimos requeridos para cada nivel educativo.

Ante la problemática de la diversidad de perfiles y como una alternativa de profesionalización del docente de matemáticas, el MTSK puede fungir como un espejo y una guía en la que los profesores de matemáticas puedan identificar sus fortalezas y áreas de oportunidad frente a aquellos contenidos en los que fueron formados o en los que incluso carecieron de toda orientación, hablándose de lo pedagógico o disciplinar.

El MTSK va acorde a las exigencias de las nuevas reformas y es una opción para que los docentes, teniendo un abordaje gradual del mismo e independientemente de la formación inicial que tuvieron, puedan tener una homogeneización tanto de saberes matemáticos como de sus prácticas de enseñanza.

\section{Fuentes de consulta}

Beneitone, P., Esquetini, C., González, J., Marty, M., Siufi, G. y Wagenaar, R. (Eds.). (2007). Reflexiones y perspectivas de la educación superior en América Latina. Informe final-Proyecto Tuning-América Latina 2004-2007. España: Universidad de Deusto/Universidad de Groningen. Recuperado de: https://bit.ly/2BoAfC7.

Flores, E., Escudero, D., Montes, M. y Aguilar, A. (2014). Nuestra modelación del conocimiento especializado del profesor de matemáticas. En J. Carrillo, L. Contreras, N. Climent, D. Escudero, E. Flores y M. Montes (Eds.), Un marco teórico para el conocimiento especializado del profesor de matemáticas, 57-72. España: Universidad de Huelva.

Hernández, J., Sosa, L. y López, I. (2013). Los formadores de profesores como punto de inflexión en la educación. En R. Ibarra, E. Bueno, R. Ibarra y J. Hernández (Coords.), Diferentes perspectivas y posibles soluciones para la crisis en América Latina, 3376-3390. México: Signo Imagen.

Pérez, C. (2017). Competencias y campos de acción presentes en los currículos oficiales para la formación inicial de profesores de matemáticas del nivel medio superior (Tesis de maestría). Zacatecas, México: Universidad Autónoma de Zacatecas.

Sosa, L., Flores-Medrano, E. y Carrillo, J. (2015). Conocimiento del profesor acerca de las características de aprendizaje del álgebra en bachillerato. Enseñanza de las Ciencias, 33.2, 173-189. 